
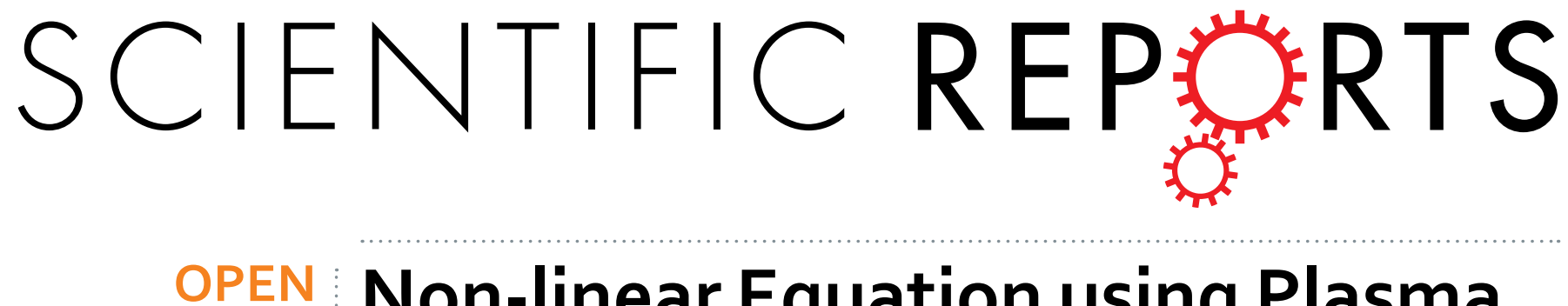

\title{
Non-linear Equation using Plasma Brain Natriuretic Peptide Levels to Predict Cardiovascular Outcomes in \\ Patients with Heart Failure
}

Received: 08 July 2016

Accepted: 24 October 2016

Published: 15 November 2016
Hiroki Fukuda ${ }^{1,2, *}$, Hideaki Suwa $^{1, *{ }^{*}+}$, Atsushi Nakano ${ }^{1,3}$, Mari Sakamoto ${ }^{1,2}$, Miki Imazu $^{1,2}$, Takuya Hasegawa ${ }^{1}$, Hiroyuki Takahama ${ }^{1}$, Makoto Amaki ${ }^{1}$, Hideaki Kanzaki ${ }^{1}$, Toshihisa Anzai ${ }^{1}$, Naoki Mochizuki ${ }^{2}$, Akira Ishii ${ }^{4}$, Hiroshi Asanuma ${ }^{3,5}$, Masanori Asakura ${ }^{1,3}$, Takashi Washio ${ }^{4}$ \& Masafumi Kitakaze ${ }^{1,3}$

Brain natriuretic peptide (BNP) is the most effective predictor of outcomes in chronic heart failure (CHF). This study sought to determine the qualitative relationship between the BNP levels at discharge and on the day of cardiovascular events in CHF patients. We devised a mathematical probabilistic model between the BNP levels at discharge $(y)$ and on the day $(t)$ of cardiovascular events after discharge for $113 \mathrm{CHF}$ patients (Protocol I). We then prospectively evaluated this model on another set of $60 \mathrm{CHF}$ patients who were readmitted (Protocol II). $\mathrm{P}(\mathrm{t} \mid \mathrm{y})$ was the probability of cardiovascular events occurring after $>t$, the probability on $t$ was given as $p(t \mid y)=-d P(t \mid y) / d t$, and $p(t \mid y)=p P(t \mid y)=\alpha y^{3} P(t \mid y)$, along with $p=\alpha y^{\beta}$ ( $\alpha$ and $\beta$ were constant); the solution was $p(t \mid y)=\alpha y^{\beta} \exp \left(-\alpha y^{\beta} t\right)$. We fitted this equation to the data set of Protocol I using the maximum likelihood principle, and we obtained the model $p(t \mid y)=0.000485 y^{0.24788} \exp \left(-0.000485 y^{0.24788} t\right)$. The cardiovascular event-free rate was computed as $P(t)=1 / 60 \Sigma_{i=1, \ldots, 60} \exp \left(-0.000485 y_{i}{ }^{0.24788} t\right)$, based on this model and the BNP levels $y_{i}$ in a data set of Protocol II. We confirmed no difference between this model-based result and the actual eventfree rate. In conclusion, the BNP levels showed a non-linear relationship with the day of occurrence of cardiovascular events in CHF patients.

Because chronic heart failure (CHF) is one of the most common fatal diseases and has an increasing prevalence worldwide ${ }^{1}$, its severity must be correctly diagnosed for the appropriate treatment to be implemented. Because it has been shown that brain natriuretic peptide (BNP) is linked to the pathophysiology of $\mathrm{HF}^{2,3}$ and that higher plasma BNP levels qualitatively predict more frequent clinical events, such as hospitalization or cardiovascular death, clinicians refer to the plasma BNP levels when diagnosing or treating CHF patients ${ }^{4,5}$. Plasma BNP levels have been recognized to correspond to the severity of CHF because of the peptide secreted from cardiac cells in response to wall stress of the heart ${ }^{6-8}$. However, BNP is known to be cardioprotective $\mathrm{e}^{9,10}$. Therefore, BNP functions to improve cardiovascular function and reflect cardiovascular dysfunction, which suggests that it is intricately and complexly linked to the pathophysiology of CHF. However, there has not been a clear consensus on whether the plasma BNP levels quantitatively predict the time of cardiovascular events in CHF patients. Furthermore, it is unclear whether the extent of BNP-level changes quantitatively predicts the interval to the occurrence of cardiovascular events. For subjects with stable CHF, increased risk is evident in patients with a BNP level of at least $100 \mathrm{pg} / \mathrm{mL}$; every increase of $100 \mathrm{pg} / \mathrm{mL}$ is associated with a $35 \%$ increase in risk ${ }^{11,12}$. However, the probability that BNP levels of $100-200 \mathrm{pg} / \mathrm{mL}$ have a comparable tendency to cause cardiovascular events as BNP

${ }^{1}$ Department of Cardiovascular Medicine, National Cerebral and Cardiovascular Center, 5-7-1 Fujishirodai, Suita, Osaka, Japan. ${ }^{2}$ Department of Cell Biology, National Cerebral and Cardiovascular Center, 5-7-1 Fujishirodai, Suita, Osaka, Japan. ${ }^{3}$ Department of Clinical Research and Development, National Cerebral and Cardiovascular Center, 5-7-1 Fujishirodai, Suita, Osaka, Japan. ${ }^{4}$ The institute of Scientific and Industrial Research, Osaka University, 1-1 Yamadaoka, Suita, Osaka, Japan. ${ }^{5}$ Department of Cardiovascular Medicine, Kyoto Prefectural University of Medicine, Kyoto, Japan. ${ }^{\dagger}$ Present address: Tochigi Medical Center, Tochigi, Japan. ${ }^{*}$ These authors contributed equally to this work. Correspondence and requests for materials should be addressed to M.K. (email: kitakaze@zf6.so-net.ne.jp) 
levels of $900-1,000 \mathrm{pg} / \mathrm{mL}$ is questionable. Based on this hypothesis, we aimed to construct an exquisite model to establish the link between the plasma BNP levels and the day of re-hospitalization because of the worsening of HF or cardiovascular death in patients with $\mathrm{HF}$.

Using our database of patients who were admitted because of acute decompensated heart failure (ADHF) and were discharged as stable CHF after sufficient treatment, we performed this study with the following objectives: (1) to theoretically derive a probability formula that correlates the plasma BNP levels at discharge and the day of hospitalization or cardiac death in patients with stable CHF; (2) to obtain an actual probability model by fitting the theoretical probability formula to the clinical data of the retrospectively enrolled patients; and (3) to prospectively test whether the cardiovascular event-free rate using this model in CHF patients was consistent with the actual cardiovascular event-free rate in the patients.

\section{Methods}

Study protocols. In Protocol I, we retrospectively enrolled CHF patients to constitute an actual probability model that would provide the day of cardiovascular events based on the plasma BNP levels alone. In Protocol II, we prospectively tested whether the expected date, as determined by the probability model, was consistent with the actual day of cardiovascular events.

Protocol I. From our database of CHF patients, 113 ADHF patients who were admitted because of worsening $\mathrm{CHF}$ and were discharged after improvement of HF to New York Health Association (NYHA) I or II between January 2007 and December 2008 were retrospectively enrolled. The follow-up period was until December 2014. For patients who were admitted to the hospital several times during the follow-up period, the earliest date of hospitalization was recorded. CHF was diagnosed by an expert team of cardiologists using the Framingham criteria ${ }^{13}$. The time of the discharge was determined by the expert team of cardiologists in charge of the HF department. Discharge was recommended when the patients presented no signs of decompensation, such as NYHA functional class $<3$, rales, and gallop rhythm; when their blood pressure was stable; and when there was an improvement in renal function after optimal treatment following international guidelines ${ }^{14}$. In each patient, the plasma BNP level at discharge was recorded. Similarly, the first day of occurrence of cardiovascular events was recorded. A cardiovascular event was defined as re-hospitalization because of worsening HF or cardiovascular death. An independent group of physicians in the emergency room at our institute made the decision on re-hospitalization when the CHF of the patients progressed and worsened; they also analysed the primary cause of re-hospitalization or death. We fitted our theoretical probability model, which is described below, to the data on the BNP levels at discharge and the day of the cardiovascular events to approximate the probability of cardiovascular events based on these BNP levels.

Protocol II. We prospectively enrolled 60 ADHF patients who were admitted because of worsening HF and were discharged after improvement of HF to NYHA I or II between May 2013 and December 2015. We followed-up these patients until the end of 2015. The primary endpoint was the day of the first re-hospitalization because of cardiovascular causes or cardiovascular death. An independent group of physicians in the emergency room of our institute made a decision on re-hospitalization when the CHF of the patients progressed and worsened; they also analysed the primary cause of re-hospitalization or death. We prospectively tested whether the predictive curves of the cardiovascular event-free rate produced by the mathematical model were significantly close to the Kaplan-Meier curves for the actual cardiovascular event-free rate during the follow-up period.

Constitution of a mathematical model to link plasma BNP levels and the day of cardiovascular events for Protocols I and II. We assumed that the CHF severity in most patients did not significantly change after discharge and proportionally reflected the daily probability of cardiovascular events (p). We chose the plasma BNP levels as the most valuable index for assessing CHF severity because BNP is produced according to the progression of $\mathrm{CHF}$ and because BNP clearance from the blood is proportional to the extent of cardioprotection. On the basis of these assumptions, we estimated that the daily production and consumption of plasma BNP were proportional to the power of $\mathrm{p}\left(\mathrm{p}^{\gamma}\right)$ and the plasma BNP level at discharge from the hospital (y), respectively. Because daily production $\left(\propto p^{\gamma}\right)$ and consumption $(\propto y)$ were considered to be almost equal in a given patient, $\mathrm{p}^{\gamma}=\theta \mathrm{y}$ holds. This equation was rewritten as:

$$
\mathrm{p}=\alpha y^{\beta}
$$

where $\alpha=\theta^{1 / \gamma}$ and $\beta=1 / \gamma$ were constant.

Second, $\mathrm{P}(\mathrm{t} \mid \mathrm{y})$ was designated as the probability that cardiovascular events did not occur before an interval $(\mathrm{t})$ having elapsed from discharge under a given $\mathrm{y}$. Then, $\mathrm{p}(\mathrm{t} \mid \mathrm{y})$ was designated as the probability of cardiovascular events on day $\mathrm{t}$ and was equal to the daily reduction of $\mathrm{P}(\mathrm{t} \mid \mathrm{y})$ (i.e., $-\mathrm{dP}(\mathrm{t} \mid \mathrm{y}) / \mathrm{dt})$. At the same time, $\mathrm{p}(\mathrm{t} \mid \mathrm{y})$ was equal to the probability of the first occurrence of a cardiovascular event on day $\mathrm{t}$, denoted as $\mathrm{pP}(\mathrm{t} \mid \mathrm{y})$, which is the probability that the event did not occur before day $t$ but occurred on day t. Accordingly, we obtained the following differential equation:

$$
\mathrm{P}(\mathrm{t} \mid \mathrm{y})=-\frac{\mathrm{dP}(\mathrm{t} \mid \mathrm{y})}{\mathrm{dt}}=\mathrm{pP}(\mathrm{t} \mid \mathrm{y})
$$

Because all patients were alive at discharge under a given $\mathrm{y}, \mathrm{P}(0 \mid \mathrm{y})=1$ holds. Then, we derived the following solution of this differential equation with Equation (1):

$$
\mathrm{P}(\mathrm{t} \mid \mathrm{y})=\exp \left(-\alpha y^{\beta} \mathrm{t}\right) \text { and } \mathrm{p}(\mathrm{t} \mid \mathrm{y})=\alpha y^{\beta} \exp \left(-\alpha y^{\beta} \mathrm{t}\right)
$$


Given a data set of Protocol I, DI $=\left\{\left(\mathrm{y}_{\mathrm{i}}, \mathrm{t}_{\mathrm{i}}\right) \mid \mathrm{i}=1, \ldots, \mathrm{N}\right\}$, the log-likelihood under this solution was represented as follows:

$$
\begin{aligned}
\operatorname{LL}(\mathrm{D}) & =\sum_{\mathrm{i}=1}^{\mathrm{N}} \log \left\{\mathrm{s}_{\mathrm{i}} \mathrm{p}\left(\mathrm{y}_{\mathrm{i}} \mathrm{t}_{\mathrm{i}}\right)+\left(1-\mathrm{s}_{\mathrm{i}}\right) \mathrm{P}\left(\mathrm{y}_{\mathrm{i}}, \mathrm{t}_{\mathrm{i}}\right)\right\} \\
& =\sum_{\mathrm{i}=1}^{\mathrm{N}} \log \left\{\mathrm{s}_{\mathrm{i}} \mathrm{p}\left(\mathrm{t}_{\mathrm{i}} \mid \mathrm{y}_{\mathrm{i}}\right) \mathrm{p}\left(\mathrm{y}_{\mathrm{i}}\right)+\left(1-\mathrm{s}_{\mathrm{i}}\right) \mathrm{P}\left(\mathrm{T}_{\mathrm{i}} \mid \mathrm{y}_{\mathrm{i}}\right) \mathrm{p}\left(\mathrm{y}_{\mathrm{i}}\right)\right\} \\
& =\sum_{\mathrm{i}=1}^{\mathrm{N}} \log \left\{\mathrm{s}_{\mathrm{i}} \alpha y_{\mathrm{i}}^{\beta} \exp \left(-\alpha y_{\mathrm{i}}^{\beta} \mathrm{t}_{\mathrm{i}}\right)+\left(1-\mathrm{s}_{\mathrm{i}}\right) \exp \left(-\alpha y_{\mathrm{i}}^{\beta} \mathrm{T}_{\mathrm{i}}\right)\right\}+\sum_{\mathrm{i}=1}^{\mathrm{N}} \log \mathrm{p}\left(\mathrm{y}_{\mathrm{i}}\right)
\end{aligned}
$$

where $s_{i}$ was equal to 1 if the clinical event of patient i occurred within the time span of observation in DI; otherwise, $s_{i}$ was equal $0 . T_{i}$ was the elapsed day at the close of the observation for patients with $s_{i}=0 . p(y, t)$ is the probability of observing a patient with a BNP level of $y$ at discharge and a cardiovascular event at day $t$ in the data set. Similarly, $\mathrm{P}(\mathrm{y}, \mathrm{T})$ is the probability of observing a patient with a BNP level of $\mathrm{y}$ at discharge and a cardiovascular event after day $\mathrm{T}$ in the data set. $\mathrm{p}(\mathrm{y})$ is the distribution of $\mathrm{y}$ in the data set. Because the second term on the last line did not depend on parameters $\alpha$ and $\beta$, we searched for the combination of these parameters that maximized the first term on the data set DI by a standard non-linear optimization algorithm named the Nelder-Mead Simplex Method ${ }^{15}$.

Under these optimum parameters obtained by DI of Protocol $\mathrm{I}$, the model-based event-free rate $(\mathrm{P}(\mathrm{t}))$ at $\mathrm{t}$ for a BNP data set of Protocol II, DII ${ }_{y}=\left\{y_{i} \mid i=1, \ldots, M\right\}$, was computed using an approximation of $p(y)$ by its data distribution in $\mathrm{DII}_{\mathrm{y}}: \sum_{\mathrm{i}=1}^{\mathrm{M}} \delta\left(\mathrm{y}_{\mathrm{i}}\right)$, where $\delta(\bullet)$ is Dirac's delta function. Because $\mathrm{P}(\mathrm{t})$ was equivalent to the probability that a cardiovascular event occurred after day $\mathrm{t}$, it was computed as follows:

$$
\begin{aligned}
\mathrm{P}(\mathrm{t}) & =\int_{\mathrm{t}}^{\infty} \int_{0}^{\infty} \mathrm{p}(\tau \mid \mathrm{y}) \mathrm{p}(\mathrm{y}) \mathrm{dyd} \tau \cong \int_{\mathrm{t}}^{\infty} \int_{0}^{\infty} \mathrm{p}(\tau \mid \mathrm{y}) \sum_{\mathrm{i}=1}^{\mathrm{M}} \delta\left(\mathrm{y}_{\mathrm{i}}\right) \mathrm{dyd} \tau \\
& =\sum_{\mathrm{i}=1}^{\mathrm{M}} \int_{\mathrm{t}}^{\infty} \mathrm{p}\left(\tau \mid \mathrm{y}_{\mathrm{i}}\right) d \tau=\sum_{\mathrm{i}=1}^{\mathrm{M}} \mathrm{P}\left(\mathrm{t} \mid \mathrm{y}_{\mathrm{i}}\right)=\sum_{\mathrm{i}=1}^{\mathrm{M}} \exp \left(-\alpha y_{\mathrm{i}}^{\beta} \mathrm{t}\right) .
\end{aligned}
$$

Moreover, the model obtained by DI enabled us to compute the event-free rate curve $\mathrm{P}(\mathrm{t})$ for a group of patients with BNP levels in a specific interval $\left(\mathrm{y}_{\mathrm{l}}, \mathrm{y}_{\mathrm{u}}\right)$ at discharge in DII. This was performed by limiting the distribution $\mathrm{p}(\mathrm{y})$ into $\left(\mathrm{y}_{\mathrm{l}}, \mathrm{y}_{\mathrm{u}}\right)$, i.e., limiting the patients to those having BNP levels in $\left(\mathrm{y}_{\mathrm{l}}, \mathrm{y}_{\mathrm{u}}\right)$ in DII $\mathrm{y}_{\mathrm{y}}$ as $\mathrm{p}\left(\mathrm{y} \mid\left(\mathrm{y}_{1} \mathrm{y}_{\mathrm{u}}\right)\right) \cong \sum_{\mathrm{Dll}_{\mathrm{y}}\left(\mathrm{y}_{\mathrm{p}}, \mathrm{y}_{\mathrm{u}}\right)} \delta\left(\mathrm{y}_{\mathrm{i}}\right)$ where the summation was taken over $\operatorname{DII}_{\mathrm{y}}\left(\mathrm{y}_{\mathrm{l}}, \mathrm{y}_{\mathrm{u}}\right)=\left\{\mathrm{y}_{\mathrm{i}} \mid \mathrm{y}_{\mathrm{l}}<\mathrm{y}_{\mathrm{i}}<\mathrm{y}_{\mathrm{u}}\right.$ in DII $\mathrm{D}_{\mathrm{y}}$. Thus, we showed an event-free rate curve $\mathrm{P}\left(\mathrm{t} \mid\left(\mathrm{y}_{\mathrm{l}}, \mathrm{y}_{\mathrm{u}}\right)\right)$ for the patient group as:

$$
\begin{aligned}
\mathrm{P}\left(t \mid\left(\mathrm{y}_{1}, \mathrm{y}_{\mathrm{u}}\right)\right) & =\int_{\mathrm{t}}^{\infty} \int_{0}^{\infty} \mathrm{p}(\tau \mid \mathrm{y}) \mathrm{p}\left(y \mid\left(\mathrm{y}_{1}, \mathrm{y}_{\mathrm{u}}\right)\right) \mathrm{dyd} \tau \\
& \cong \int_{\mathrm{t}}^{\infty} \int_{0}^{\infty} \mathrm{p}(\tau \mid \mathrm{y}) \sum_{\operatorname{Dll}_{\mathrm{y}}\left(\mathrm{y}_{1}, \mathrm{y}_{\mathrm{u}}\right)} \delta\left(\mathrm{y}_{\mathrm{i}}\right) \mathrm{dyd} \tau \\
& =\sum_{\operatorname{Dll}_{\mathrm{y}}\left(\mathrm{y}_{1}, \mathrm{y}_{\mathrm{u}}\right)} \int_{\mathrm{t}}^{\infty} \mathrm{p}\left(\tau \mid \mathrm{y}_{\mathrm{i}}\right) d \tau=\sum_{\operatorname{Dll}_{\mathrm{y}}\left(\mathrm{y}_{1}, \mathrm{y}_{\mathrm{u}}\right)} \mathrm{P}\left(\mathrm{t} \mid \mathrm{y}_{\mathrm{i}}\right)=\sum_{\operatorname{Dll}_{\mathrm{y}}\left(\mathrm{y}_{1}, \mathrm{y}_{\mathrm{u}}\right)} \exp \left(-\alpha y_{\mathrm{i}}^{\beta} \mathrm{t}\right)
\end{aligned}
$$

Ethics Statement. Both Protocols I and II were approved by the National Cerebral and Cardiovascular Centre Research Ethics Committee. For Protocol I, the committee decided that the acquisition of informed consent from the 113 subjects was not required, according to the Japanese Clinical Research Guidelines and because this was a retrospective observational study. Instead, we made a public announcement on both the Internet homepage of our institution and the bulletin boards of our outpatient and inpatient clinics, in accordance with the request of the ethics committee and the guidelines. In Protocol II, written informed consent was obtained from all 60 subjects (UMIN000018691). This was a clinical study that enrolled patients with CHF, and all methods were performed in accordance with the relevant guidelines and regulations.

Statistical Analysis. Normally distributed data are expressed as the mean \pm standard deviation; other values were reported as median and interquartile range (IQR). We performed the goodness-of-fit test and used the coefficient of determination as a measure to assess the significant relationships between the predictive curves and actual Kaplan-Meier curves of the cardiovascular event-free rate. The differences in the predictive curves among the different BNP levels were tested using the Wilcoxon signed-rank test. We estimated the error bounds of the parameters $\alpha$ and $\beta$ by applying the standard bootstrap sampling ${ }^{16}$ to the data set DI of Protocol I, and we added the upper and lower $2 \sigma$ bound lines of the model based Kaplan-Meier curves obtained in Protocol II. All tests were two-tailed, and $\mathrm{P}<0.05$ was considered significant. All analyses were performed using JMP software for Windows (version 8.0.2, SAS Inc., Cary, NC, USA).

\section{Results}

In Protocol I, a total of $113 \mathrm{HF}$ patients (71 men and 42 women, median age of 67 years) were retrospectively enrolled in this study (Table 1). All patients were in NYHA functional class I or II upon discharge. The data on the plasma BNP levels at discharge were used to obtain a probability model for the day of cardiovascular events because it approximated the function of plasma BNP. We fitted the model to the data on the plasma BNP levels 


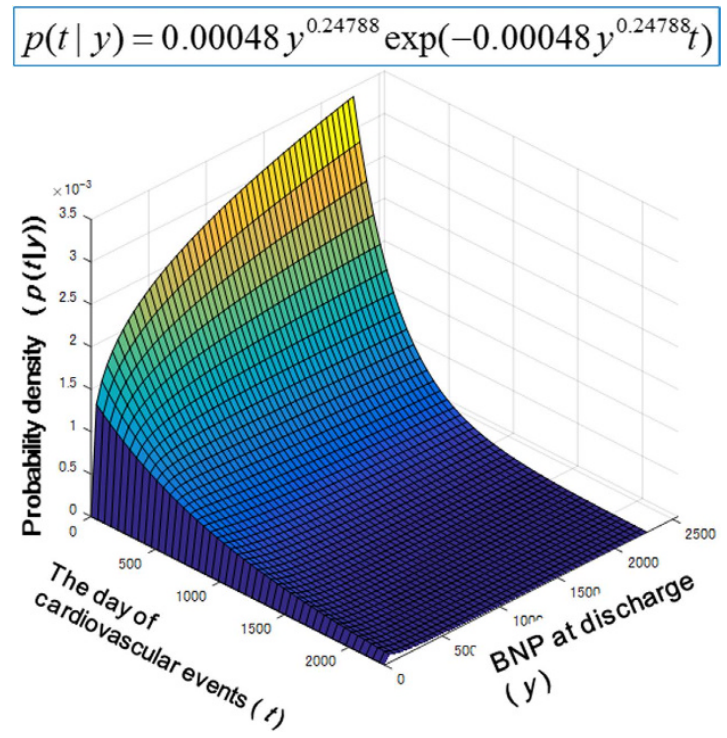

Figure 1. Fitting of the actual data on the plasma BNP levels and the day of cardiovascular events to the theoretical equation model for patients with ADHF. This shows the conditional probability of the elapsed date at a clinical event after discharge $(\mathrm{t})$ under a given BNP level at discharge (y).

at discharge and the day of cardiovascular events by using the maximization of the aforementioned logarithmic likelihood LL(D). We obtained the parameters of $\alpha=0.000485$ and $\beta=0.24788$ to characterize the daily probability of the cardiovascular event as equation (1) at the optimum. Accordingly, we obtained the probability model $\mathrm{p}(\mathrm{t} \mid \mathrm{y})=0.000485 \mathrm{y}^{0.24788} \exp \left(-0.000485 \mathrm{y}^{0.24788} \mathrm{t}\right)$ (Fig. 1$)$.

For Protocol II, the patients' characteristics are shown in Table 2. Figure 2 shows the Kaplan-Meier plots calculated by using the actual data and the model for cardiovascular event-free periods. Both lines are significantly close to each other $\left(\mathrm{R}^{2}=0.92\right)$. The upper and lower $2 \sigma$ bound lines of the model plot did not completely overlap with the actual Kaplan-Meier curve because the actual Kaplan-Meier curves also fluctuated due to their statistical uncertainty. Figure 3A-C show the Kaplan-Meier plots of the actual data and the model for the cardiovascular event-free period in each group, with plasma BNP levels $\leq 200 \mathrm{pg} / \mathrm{mL}(\mathrm{n}=16), 200 \mathrm{pg} / \mathrm{mL}<\mathrm{BNP} \leq 500 \mathrm{pg} / \mathrm{mL}$ $(\mathrm{n}=21)$ and BNP levels $>500 \mathrm{pg} / \mathrm{mL}(\mathrm{n}=23)$. The $2 \sigma$ bounds of the model-based curves are also provided. In each group, both lines are significantly close to each other $\left(\mathrm{R}^{2}=0.94,0.90\right.$ and 0.95 for Fig. 3A-C). Figure 3D depicts the Kaplan-Meier plots of the model-based event-free rates and shows that the plots become steeper when the BNP levels increase.

\section{Discussion}

Plasma BNP levels are known to be related to the probability of cardiovascular events, such as hospitalization or cardiovascular death. However, there is no clear consensus on whether the plasma BNP levels quantitatively predict the time of occurrence of cardiovascular events. In the present study, we constituted a theoretical probability model to estimate the time of cardiovascular event occurrence based on the plasma BNP levels at discharge in CHF patients who were admitted to our hospital because of ADHF. We provided a non-linear probability model, $\mathrm{p}(\mathrm{t} \mid \mathrm{y})=0.000485 \mathrm{y}^{0.24788} \exp \left(-0.000485 \mathrm{y}^{0.24788} \mathrm{t}\right)$, and a model-based formula of the event-free rate, $\mathrm{P}(\mathrm{t}) \cong \sum_{\mathrm{i}=1}^{\mathrm{M}} \exp \left(-0.000485 \mathrm{y}_{\mathrm{i}}^{0.24788} \mathrm{t}\right)$, by fitting the actual data on the BNP levels and the day of cardiovascular events. We further validated the accuracy and reproducibility of this model in a prospective observational study and found that the model provided a plausible probability of a given BNP level to assess the time of occurrence of cardiovascular events. As shown in Fig. 3, the calculated and actual cardiovascular event-free rates are well correlated with each other, although the times of the beginning and end of cardiovascular events were slightly separate. This figure is comparable to the data from the Seattle Heart Failure Model ${ }^{17}$; however, our model is derived from only the BNP levels at discharge based on the pathophysiological assumption of BNP metabolism and by fitting the obtained equation to the actual data and validating the obtained equation using $\mathrm{CV}$ and another ADHF cohort, whereas Levy et al. obtained the predicted equation by fitting the survival to many factors using multivariate analysis and validating it with another cohort of $\mathrm{CHF}^{17}$.

$\mathrm{BNP}$ is produced in cardiomyocytes by both ventricular stress and excess neurohumoral factors ${ }^{6-8}$. Because the LV mass is larger than the RV mass, the plasma BNP levels primarily reflect LV dysfunction. Plasma BNP appears to be cardioprotective by increasing cyclic GMP levels and activating G kinase ${ }^{10}$, but it also increases with worsening $\mathrm{HF}^{18}$; these facts provide evidence that increases in the BNP levels may precisely predict the occurrence of cardiovascular events in patients with HF. Indeed, the plasma BNP levels were shown to decrease after treatment modalities that are known to improve the clinical outcomes of patients with $\mathrm{CHF}^{19}$. This suggests that BNP not only is a biomarker of the severity of cardiac dysfunction but also is linked to cardioprotection. Therefore, the BNP levels may precisely predict the clinical outcomes in CHF patients. 


\begin{tabular}{|c|c|}
\hline & Population $(n=113)$ \\
\hline Age, yrs & $63.6 \pm 16.2$ \\
\hline Male & $70(62)$ \\
\hline \multicolumn{2}{|l|}{ Cause of ADHF } \\
\hline Cardiomyopathy & $45(40)$ \\
\hline Hypertensive heart disease & $13(12)$ \\
\hline Ischemic heart disease & $12(11)$ \\
\hline Valvular heart disease & $26(23)$ \\
\hline Cardiac sarcoidosis & $4(3)$ \\
\hline Myocarditis & $1(1)$ \\
\hline Others & $12(11)$ \\
\hline \multicolumn{2}{|l|}{ Comorbidity } \\
\hline Hypertension & $45(40)$ \\
\hline Diabetes mellitus & $32(28)$ \\
\hline Hyperlipidemia & $25(22)$ \\
\hline Chronic AF & $25(22)$ \\
\hline \multicolumn{2}{|l|}{ Signs at admission } \\
\hline Elevated jugular venous pressure & $55(49)$ \\
\hline $\mathrm{S}_{3}$ gallop & $71(63)$ \\
\hline Lower extremity edema & $63(56)$ \\
\hline NYHA functional class: III/IV & $75(66) / 38(34)$ \\
\hline \multicolumn{2}{|l|}{ Baseline characteristics at admission/at discharge } \\
\hline Heart rate, beats/min & $83 \pm 26 / 66 \pm 10$ \\
\hline Systolic BP, mmHg & $120 \pm 31 / 105 \pm 15$ \\
\hline Diastolic BP, mmHg & $69 \pm 17 / 59 \pm 7$ \\
\hline Body weight, kg & $58.8 \pm 13.4 / 53.3 \pm 12.1$ \\
\hline \multicolumn{2}{|l|}{ Laboratory factors at discharge } \\
\hline Hemoglobin, g/dl & $12.2 \pm 2.1$ \\
\hline Blood urea nitrogen, mg/dl & $26.0 \pm 11.9$ \\
\hline Creatinine, $\mathrm{mg} / \mathrm{dl}$ & $1.13 \pm 0.56$ \\
\hline Sodium, mEq/L & $136.8 \pm 4.0$ \\
\hline Uric acid, $\mathrm{mg} / \mathrm{dl}$ & $7.2 \pm 2.2$ \\
\hline C-reactive protein, $\mathrm{mg} / \mathrm{dl}$ & $0.5 \pm 1.3$ \\
\hline BNP at admission/at discharge, median (IQR), $\mathrm{pg} / \mathrm{ml}$ & $626(276-1123) / 244(125-397)$ \\
\hline \multicolumn{2}{|l|}{ Echocardiographic factors at admission/at discharge } \\
\hline Left ventricular end-diastolic dimension, $\mathrm{mm}$ & $58.8 \pm 13.2 / 58.9 \pm 12.5$ \\
\hline Left ventricular end-systolic dimension, $\mathrm{mm}$ & $46.5 \pm 15.4 / 46.3 \pm 15.7$ \\
\hline Fractional shortening, $\%$ & $22.4 \pm 12.2 / 23.2 \pm 12.6$ \\
\hline Left atrial diastolic dimension, $\mathrm{mm}$ & $51.0 \pm 9.2 / 48.4 \pm 8.6$ \\
\hline Pressure across tricuspid valve, $\mathrm{mmHg}$ & $38.5 \pm 15.0 / 28.2 \pm 12.3$ \\
\hline \multicolumn{2}{|l|}{ Medication at admission } \\
\hline Use of dopamine & $6(4)$ \\
\hline Use of dobutamine & $28(25)$ \\
\hline Use of phosphodiesterase inhibitor & $16(14)$ \\
\hline Use of hANP & $23(20)$ \\
\hline Use of nitroglycerin & $14(12)$ \\
\hline Use of intravenous diuretics & $37(33)$ \\
\hline \multicolumn{2}{|l|}{ Medication at discharge } \\
\hline ACEi & $46(41)$ \\
\hline ARB & $33(29)$ \\
\hline Beta-blockers & $80(71)$ \\
\hline Digitalis & $38(34)$ \\
\hline Diuretics & $99(88)$ \\
\hline
\end{tabular}

Table 1. Patient characteristics of the Protocol I. Values are mean $\pm S D$ or $n(\%)$ unless otherwise indicated. IQR, interquartile range; ADHF, acute decompensated heart failure; AF, atrial fibrillation; NYHA, New York Heart Association; BNP, brain natriuretic peptide; BP, blood pressure; hANP, human atrial natriuretic peptide; $\mathrm{ACEi}$, angiotensin converting enzyme inhibitor; ARB, angiotensin II receptor blocker. 


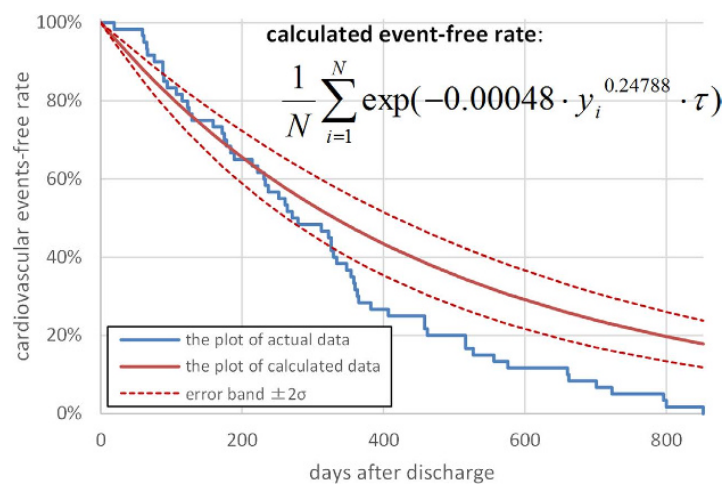

Figure 2. The Kaplan-Meier plots of calculated and actual cardiovascular event-free rate in Protocol II. The actual cardiovascular events started slightly later than the calculated events and ended earlier than the calculated events; however, the goodness-of-fit model found that KM and predictive curves were significantly close, and the coefficient of determination was 0.92 .

A
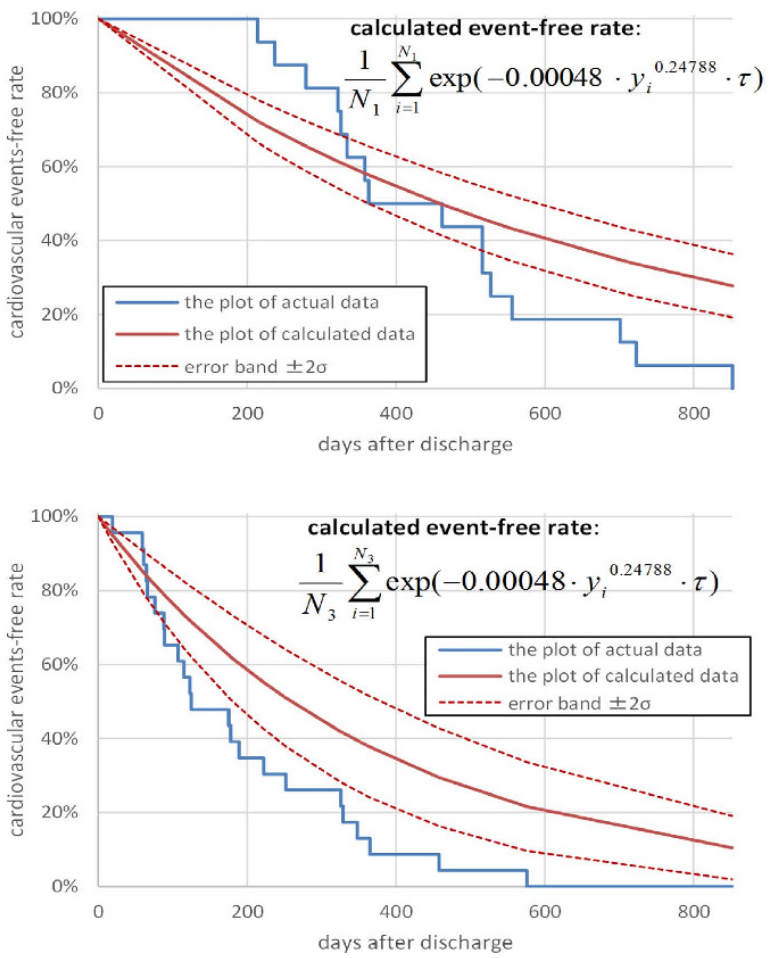

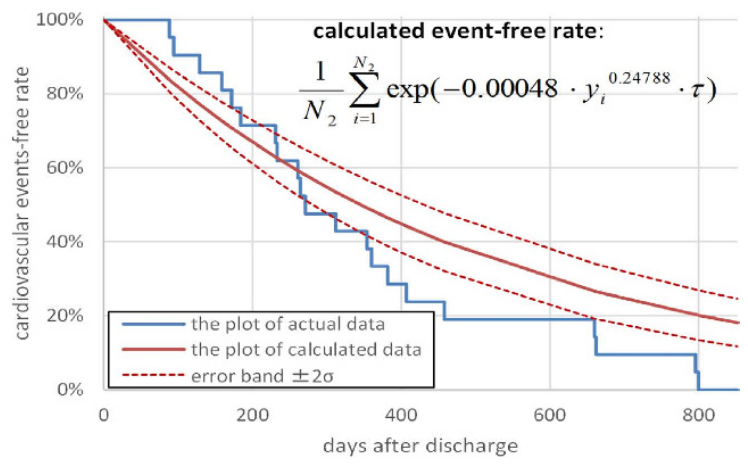

D

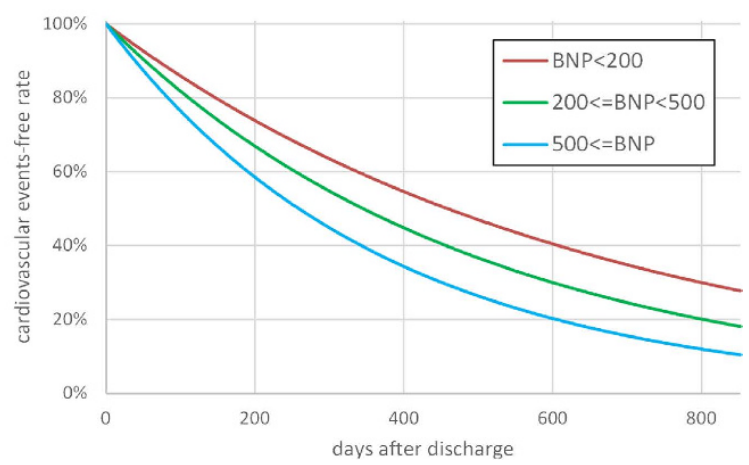

Figure 3. The Kaplan-Meier plots of calculated and actual cardiovascular event-free rate in patients with plasma BNP levels $\leq 200 \mathrm{pg} / \mathrm{mL}$ (A), $200 \mathrm{pg} / \mathrm{mL}<\mathrm{BNP} \leq 500 \mathrm{pg} / \mathrm{mL}$ (B) and BNP levels $>500 \mathrm{pg} / \mathrm{mL}$ (C). These two lines in each group are significantly close, and the coefficients of determination in $\mathbf{A}-\mathbf{C}$ are $0.94,0.90$ and 0.96 , respectively. Figure (D) shows Kaplan-Meier plots of three calculated cardiovascular events-free rates in the sets of the patients with the plasma BNP levels $\leq 200 \mathrm{pg} / \mathrm{mL}, 200 \mathrm{pg} / \mathrm{mL}<\mathrm{BNP} \leq 500 \mathrm{pg} / \mathrm{mL}$ and BNP levels $>500 \mathrm{pg} / \mathrm{mL}$.

These characteristics of BNP are different from those of other simple biological or physiologic markers of HF. For example, LV ejection fraction (LVEF) is one of the physiologic markers of HF, but treatment modalities, such as inotropic agents, that increase LVEF are not necessarily beneficial for HF. However, BNP is important for a biomarker-guided study ${ }^{19}$, which suggests that it is a more important biomarker for the prediction of cardiovascular events.

Why can the plasma BNP levels at discharge predict the probability of cardiovascular events? It does not seem to be convincing that a one-time measurement of the plasma BNP level at discharge in each HF patient can predict the day of occurrence of cardiovascular events because the BNP levels may fluctuate weekly or monthly during outpatient follow-up, despite the constant intake of medications. These changes in the BNP levels may be attributed to daily changes in hemodynamic parameters, such as heart rate or blood pressure; excessive intake of water or salt; or temporal sympathetic activation in a patient ${ }^{20}$. These facts suggest that the BNP levels at 


\begin{tabular}{|c|c|}
\hline & Population $(n=60)$ \\
\hline Age, yrs & $65.9 \pm 17.4$ \\
\hline Male & $38(63)$ \\
\hline \multicolumn{2}{|l|}{ Cause of ADHF } \\
\hline Cardiomyopathy & $26(43)$ \\
\hline Hypertensive heart disease & $5(8)$ \\
\hline Ischemic heart disease & $10(17)$ \\
\hline Valvular heart disease & $10(17)$ \\
\hline Cardiac sarcoidosis & $1(2)$ \\
\hline Myocarditis & $1(2)$ \\
\hline Others & $7(12)$ \\
\hline \multicolumn{2}{|l|}{ Comorbidity } \\
\hline Hypertension & $29(48)$ \\
\hline Diabetes mellitus & $21(35)$ \\
\hline Hyperlipidemia & $20(33)$ \\
\hline Chronic AF & $26(43)$ \\
\hline \multicolumn{2}{|l|}{ Signs at admission } \\
\hline Elevated jugular venous pressure & $28(47)$ \\
\hline $\mathrm{S}_{3}$ gallop & $34(57)$ \\
\hline Lower extremity edema & $41(68)$ \\
\hline NYHA functional class: III/IV & $35(58) / 25(42)$ \\
\hline \multicolumn{2}{|l|}{ Baseline characteristics at admission/at discharge } \\
\hline Heart rate, beats/min & $82 \pm 19 / 66 \pm 9$ \\
\hline Systolic BP, mmHg & $123 \pm 23 / 107 \pm 20$ \\
\hline Diastolic BP, mmHg & $70 \pm 16 / 61 \pm 17$ \\
\hline Body weight, kg & $60.8 \pm 15.2 / 56.2 \pm 14.5$ \\
\hline \multicolumn{2}{|l|}{ Laboratory factors at discharge } \\
\hline Hemoglobin, g/dl & $12.1 \pm 2.4$ \\
\hline Blood urea nitrogen, mg/dl & $39.0 \pm 27.4$ \\
\hline Creatinine, $\mathrm{mg} / \mathrm{dl}$ & $1.09 \pm 0.40$ \\
\hline Sodium, mEq/L & $138.6 \pm 5.3$ \\
\hline Uric acid, $\mathrm{mg} / \mathrm{dl}$ & $6.7 \pm 2.4$ \\
\hline C-reactive protein, $\mathrm{mg} / \mathrm{dl}$ & $1.1 \pm 2.0$ \\
\hline BNP at admission/at discharge, median (IQR), $\mathrm{pg} / \mathrm{ml}$ & $963(754-1366) / 377(198-816)$ \\
\hline \multicolumn{2}{|l|}{ Echocardiographic factors at admission/at discharge } \\
\hline Left ventricular end-diastolic dimension, $\mathrm{mm}$ & $61.0 \pm 13.5 / 62.0 \pm 14.3$ \\
\hline Left ventricular end-systolic dimension, $\mathrm{mm}$ & $51.2 \pm 16.6 / 51.7 \pm 17.3$ \\
\hline Fractional shortening, $\%$ & $17.8 \pm 12.2 / 18.3 \pm 12.2$ \\
\hline Left atrial diastolic dimension, $\mathrm{mm}$ & $51.7 \pm 7.8 / 47.8 \pm 7.6$ \\
\hline Pressure across tricuspid valve, $\mathrm{mmHg}$ & $40.1 \pm 8.5 / 30.3 \pm 6.8$ \\
\hline \multicolumn{2}{|l|}{ Medication at admission } \\
\hline Use of dopamine & $3(5)$ \\
\hline Use of dobutamine & $12(20)$ \\
\hline Use of phosphodiesterase inhibitor & $10(17)$ \\
\hline Use of hANP & $21(35)$ \\
\hline Use of nitroglycerin & $15(25)$ \\
\hline Use of intravenous diuretics & $23(38)$ \\
\hline \multicolumn{2}{|l|}{ Medication at discharge } \\
\hline ACEi & $28(47)$ \\
\hline ARB & $9(15)$ \\
\hline Beta-blockers & $48(80)$ \\
\hline Digitalis & $18(30)$ \\
\hline Diuretics & $55(92)$ \\
\hline
\end{tabular}

Table 2. Patient characteristics of the Protocol II. Values are mean \pm SD or $\mathrm{n}(\%)$ unless otherwise indicated. IQR, interquartile range; ADHF, acute decompensated heart failure; AF, atrial fibrillation; NYHA, New York Heart Association; BNP, brain natriuretic peptide; BP, blood pressure; hANP, human atrial natriuretic peptide; $\mathrm{ACEi}$, angiotensin converting enzyme inhibitor; $\mathrm{ARB}$, angiotensinII receptor blocker. 


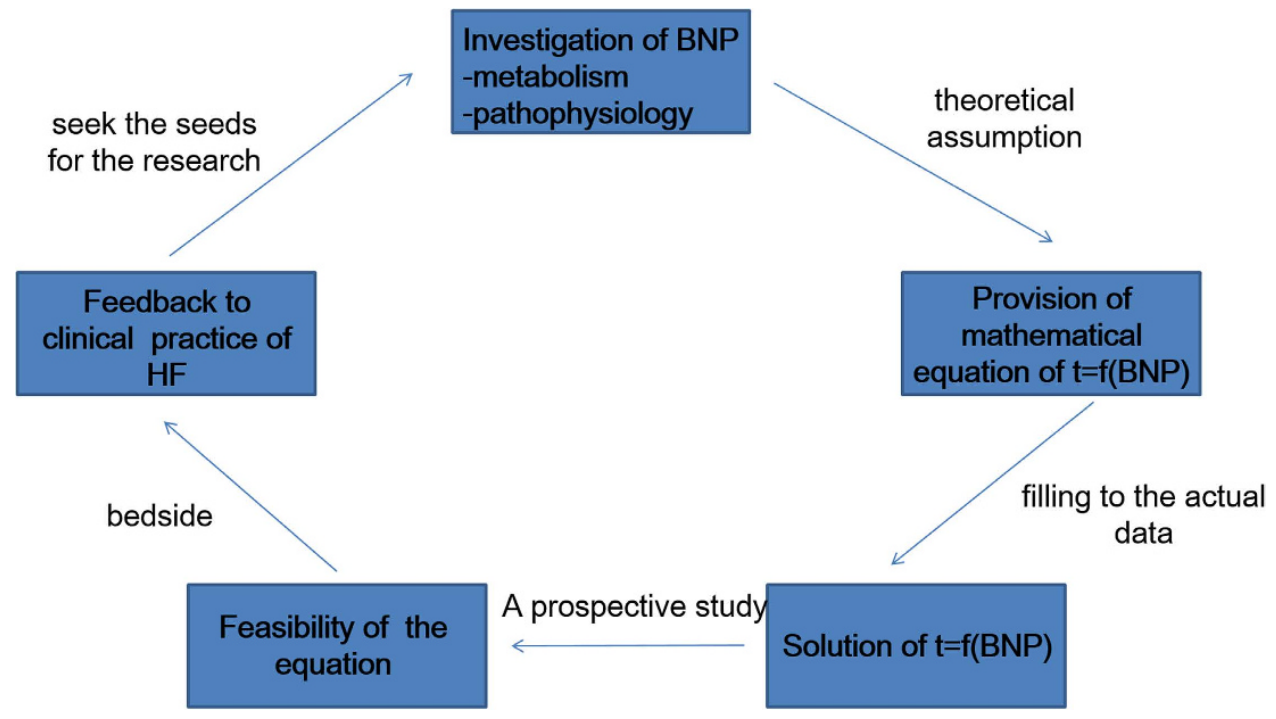

Figure 4. The schema and procedure to provide the mathematical probabilistic model between plasma BNP levels and the day of cardiovascular events. BNP, brain natriuretic peptide; HF, heart failure.

discharge cannot determine the fate of the CHF patients. However, CHF patients who are discharged from the hospital receive the ideal medications, medical apparatus, water/salt balance, and haemodynamics; this implies that patients are in the best condition at discharge, thus providing the lowest value of BNP levels for that patient. Indeed, in this study, the plasma BNP level at admission decreased from 917 to $351 \mathrm{pg} / \mathrm{mL}$ after comprehensive treatment for ADHF (Table 1). Such BNP levels may be considered to represent the best clinical condition of the patient and may reflect the true severity of CHF and the probability of cardiovascular events.

Indeed, reports have shown that the pre-discharge BNP or NT-pro BNP levels that were measured only during hospitalization predicted clinical outcomes ${ }^{21,22}$. This BNP value at the best clinical condition may affect the probability of the clinical outcomes. Of course, the actual and baseline cardiac function may worsen during follow-up; we cannot deny this possibility, and this deviation may cause the actual day of occurrence of cardiovascular events to deviate from the estimated days.

$\mathrm{BNP}$ in plasma has been used as a sole biomarker for CHF and is recognized to precisely predict cardiovascular events. However, specialized medical care has been focused because many clinical, social, and genomic factors are involved in patients with $\mathrm{CHF}^{23}$. However, at the same time, we should seek the most important factor to ascertain the disease and investigate the precise relationship between that factor and the clinical outcomes. This method of investigation that uses BNP as a biomarker for CHF may also contribute to the choice of an appropriate medicine.

The most important information of the present study was that the increase in the risk for re-hospitalization was not proportional or linear to the increase in BNP levels for subjects with stable CHF following ADHF, even if the subsequent BNP levels were more than $100 \mathrm{pg} / \mathrm{mL}$, although every $100 \mathrm{pg} / \mathrm{mL}$ rise was associated with a $35 \%$ increase in risk for subjects with stable CHF. The probability of occurrence of clinical outcomes in patients with $\mathrm{CHF}$ was related to the BNP levels at a power of approximately $1 / 4$. We do not know how the changes in the BNP levels correspond to the quantitative improvement or worsening of $\mathrm{CHF}$, and we believe that one of the advantages of the present study is that we can propose such an equation to yield quantitative meanings for the $\mathrm{BNP}$ values. It would be better to transform the BNP levels to $1 / \mathrm{BNP}^{0.25}$ to obtain an index of CHF severity from the viewpoint of clinical events. The other impact of the present study is how to employ mathematical science in clinical practice. Figure 4 shows the scenario of the present study, which aimed to both provide a theoretical equation that can be fitted to actual retrospective clinical data and evaluate the equation in a prospective study.

Limitations. The present study had several limitations. First, because this was a single-centre study, the number of patients was limited, particularly that of subjects with ADHF. Second, the quantity of data was somewhat small. However, we obtained the probability equation for 113 patients in a retrospective study and provided evidence for the plausibility of this equation in 60 patients in a prospective study. In this sense, we suggest testing the present hypothesis using multicentre trials.

\section{Conclusions.}

Our study showed that the plasma BNP levels non-linearly predicted the day of occurrence of clinical events in patients with CHF. By using our equation, the impact of BNP levels on cardiovascular outcomes may be quantitatively evaluated.

\section{References}

1. Go, A. S. et al. Heart disease and stroke statistics-2013 update: a report from the American Heart Association. Circulation 127, e6-e245, doi: 10.1161/CIR.0b013e31828124ad (2013). 
2. Yasue, H. et al. Localization and mechanism of secretion of B-type natriuretic peptide in comparison with those of A-type natriuretic peptide in normal subjects and patients with heart failure. Circulation 90, 195-203, doi: 10.1161/01.cir.90.1.195 (1994).

3. Maeda, K., Tsutamoto, T., Wada, A., Hisanaga, T. \& Kinoshita, M. Plasma brain natriuretic peptide as a biochemical marker of high left ventricular end-diastolic pressure in patients with symptomatic left ventricular dysfunction. Am. Heart J. 135, 825-832, doi: http://dx.doi.org/10.1016/S0002-8703(98)70041-9 (1998).

4. Troughton, R., Michael Felker, G. \& Januzzi, J. L. Jr. Natriuretic peptide-guided heart failure management. European heart journal 35, 16-24, doi: 10.1093/eurheartj/eht463 (2014).

5. Jourdain, P. et al. Plasma brain natriuretic peptide-guided therapy to improve outcome in heart failure: the STARS-BNP Multicenter Study. Journal of the American College of Cardiology 49, 1733-1739, doi: 10.1016/j.jacc.2006.10.081 (2007).

6. Iwanaga, Y. et al. B-type natriuretic peptide strongly reflects diastolic wall stress in patients with chronic heart failure: comparison between systolic and diastolic heart failure. J. Am. Coll. Cardiol. 47, 742-748, doi: 10.1016/j.jacc.2005.11.030 (2006).

7. Harada, M. et al. Significance of ventricular myocytes and nonmyocytes interaction during cardiocyte hypertrophy: evidence for endothelin-1 as a paracrine hypertrophic factor from cardiac nonmyocytes. Circulation 96, 3737-3744 (1997).

8. Kuwahara, K. et al. Involvement of Cardiotrophin-1 in Cardiac Myocyte-Nonmyocyte Interactions During Hypertrophy of Rat Cardiac Myocytes In Vitro. Circulation 100, 1116-1124, doi: 10.1161/01.cir.100.10.1116 (1999).

9. Nishikimi, T., Maeda, N. \& Matsuoka, H. The role of natriuretic peptides in cardioprotection. Cardiovascular research 69, 318-328, doi: 10.1016/j.cardiores.2005.10.001 (2006).

10. Levin, E. R., Gardner, D. G. \& Samson, W. K. Natriuretic Peptides. N. Engl. J. Med. 339, 321-328, doi: 10.1056/ NEJM199807303390507 (1998)

11. Doust, J. A., Pietrzak, E., Dobson, A. \& Glasziou, P. How well does B-type natriuretic peptide predict death and cardiac events in patients with heart failure: systematic review. BMJ (Clinical research ed.) 330, 625, doi: 10.1136/bmj.330.7492.625 (2005).

12. Masson, S. et al. Direct comparison of B-type natriuretic peptide (BNP) and amino-terminal proBNP in a large population of patients with chronic and symptomatic heart failure: the Valsartan Heart Failure (Val-HeFT) data. Clinical chemistry 52, 1528-1538, doi: 10.1373/clinchem.2006.069575 (2006).

13. McKee, P. A., Castelli, W. P., McNamara, P. M. \& Kannel, W. B. The natural history of congestive heart failure: the Framingham study. The New England journal of medicine 285, 1441-1446, doi: 10.1056/nejm197112232852601 (1971).

14. Hunt, S. A. et al. Focused update incorporated into the ACC/AHA 2005 Guidelines for the Diagnosis and Management of Heart Failure in Adults A Report of the American College of Cardiology Foundation/American Heart Association Task Force on Practice Guidelines Developed in Collaboration With the International Society for Heart and Lung Transplantation. Journal of the American College of Cardiology 53, e1-e90, doi: 10.1016/j.jacc.2008.11.013 (2009).

15. Avriel, M. Nonlinear programming: analysis and methods. (Dover, 2003).

16. Davison, A. C. \& Hinkley, D. V. Bootstrap Methods and their Applications. (Chambridge University Press, 1997)

17. Levy, W. C. et al. The Seattle Heart Failure Model: prediction of survival in heart failure. Circulation 113, 1424-1433, doi: 10.1161/ circulationaha.105.584102 (2006)

18. Januzzi, J. L. \& Troughton, R. Are serial BNP measurements useful in heart failure management? Serial natriuretic peptide measurements are useful in heart failure management. Circulation 127, 500-507; discussion 508, doi: 10.1161/ CIRCULATIONAHA.112.120485 (2013).

19. Felker, G. M., Hasselblad, V., Hernandez, A. F. \& O’Connor, C. M. Biomarker-guided therapy in chronic heart failure: a metaanalysis of randomized controlled trials. American heart journal 158, 422-430, doi: 10.1016/j.ahj.2009.06.018 (2009).

20. Benes, J. et al. Resting heart rate and heart rate reserve in advanced heart failure have distinct pathophysiologic correlates and prognostic impact: a prospective pilot study. JACC. Heart failure 1, 259-266, doi: 10.1016/j.jchf.2013.03.008 (2013).

21. Noveanu, M. et al. Direct comparison of serial B-type natriuretic peptide and NT-proBNP levels for prediction of short- and longterm outcome in acute decompensated heart failure. Critical care (London, England) 15, R1, doi: 10.1186/cc9398 (2011).

22. Logeart, D. et al. Predischarge B-type natriuretic peptide assay for identifying patients at high risk of re-admission after decompensated heart failure. Journal of the American College of Cardiology 43, 635-641, doi: 10.1016/j.jacc.2003.09.044 (2004).

23. Yoshida, A. et al. Derivation of a mathematical expression for predicting the time to cardiac events in patients with heart failure: a retrospective clinical study. Hypertension research: official journal of the Japanese Society of Hypertension 36, 450-456, doi: 10.1038/ hr.2012.200 (2013).

\section{Acknowledgements}

This work was supported by the Practical Research Project for Life-Style related Diseases including Cardiovascular Diseases and Diabetes Mellitus from Japan Agency for Medical Research and Development, AMED, Intramural Research Found (22-1-8,27-1-4) for Cardiovascular Diseases of National Cerebral and Cardiovascular Center and Grants from the Japan Heart Foundation for M.Kitakaze.

\section{Author Contributions}

T.W., A.I. performed statistical analysis. M.K. handled funding and supervision. H.F, H.S., A.N., M.S., M.I., T.H., H.T., M.A., H.K., T.A. acquired the data. H.F., A.N., M.K. conceived and designed the research. H.A., N.M., M.A. drafted the manuscript. M.K. made critical revision of the manuscript for key intellectual content.

\section{Additional Information}

Competing financial interests: Relationships to industry do not exist for H.F., H.S., A.N., M.S., M.I., H.T., M.A., H.K., T.A., N.M., A.I., H.A., and T.W. T.H. reports personal fees from Takeda, Daiichi-Sankyo, Otsuka, Bayer, Tanabe-Mitsubishi, Abbott Vascular Japan, Shionogi. M.A. reports grants from government during the conduct of the study; personal fees and non-financial support from Pfizer, personal fees from BoeringerIngerheim, Tanabe-Mitsubishi, Kowa and Takeda. M.K. reports grants from Japanese government, grants from Japan Heart Foundation, grants from Japan Cardiovascular Research Foundation, grants and personal fees from Takeda, Astellas, Sanofi, Pfizer, Novartis, Bheringer-Ingerheim, Tanabe-mitubishi, Kyowa-hakko-kirin, Abott, and Otsuka outside the submitted work; personal fees from Daiichi-sankyo, Ono, Bayer, from Kowa, Dainihonsumitomo, Sawai, MSD, Calpis, Shionogi, Astrazeneca, Asahikasei Med., Novo Nordisk, Fuji-film RI, and Japan Medical Data, outside the submitted work; grants from Nihon Kohden.

How to cite this article: Fukuda, H. et al. Non-linear Equation using Plasma Brain Natriuretic Peptide Levels to Predict Cardiovascular Outcomes in Patients with Heart Failure. Sci. Rep. 6, 37073; doi: 10.1038/srep37073 (2016). 
Publisher's note: Springer Nature remains neutral with regard to jurisdictional claims in published maps and institutional affiliations.

(c) (i) This work is licensed under a Creative Commons Attribution 4.0 International License. The images or other third party material in this article are included in the article's Creative Commons license, unless indicated otherwise in the credit line; if the material is not included under the Creative Commons license, users will need to obtain permission from the license holder to reproduce the material. To view a copy of this license, visit http://creativecommons.org/licenses/by/4.0/

(C) The Author(s) 2016 